This is the post-print version of the following article: Rodal-Cedeira, $S$; Vázquez Arias, $A$; Bodelon, G; Skorikov, A; Núñez-Sanchez, S; La Porta, A; Polavarapu, L; Bals, S; Liz-Marzán, LM; Perez-Juste, J; Pastoriza-Santos, I. An Expanded Surface-Enhanced Raman Scattering Tags Library by Combinatorial Encapsulation of Reporter Molecules in Metal Nanoshells ACS Nano, 2020 DOI: 10.1021/acsnano.0c04368

This article may be used for non-commercial purposes in accordance with ACS Terms and Conditions for Self-Archiving. 


\title{
An Expanded Surface-Enhanced Raman Scattering Tags Library by Combinatorial Encapsulation of Reporter Molecules in Metal Nanoshells
}

Sergio Rodal-Cedeira, ${ }^{a, b}$ Alba Vázquez-Arias, ${ }^{a, b}$ Gustavo Bodelón, ${ }^{a, b}$ Alexander Skorikov, ${ }^{c}$ Sara NuñezSánchez, ${ }^{\text {a,b }}$ Andrea Laporta, ${ }^{c}$ Lakshminarayana Polavarapu, a Sara Bals, ${ }^{c}$ Luis M. Liz-Marzán, d,e,f Jorge Pérez-Juste ${ }^{\mathrm{a}, \mathrm{b},{ }^{*}}$ and Isabel Pastoriza-Santos ${ }^{\mathrm{a}, \mathrm{b},{ }^{*}}$

a CINBIO, Universidade de Vigo, Departamento de Química Física, Campus Universitario As Lagoas, Marcosende, 36310 Vigo, Spain

b Galicia Sur Health Research Institute (IIS Galicia Sur), SERGAS-UVIGO, 36310 Vigo, Spain

c EMAT, University of Antwerp, Groenenborgerlaan 171, B-2020 Antwerp, Belgium

d CIC biomaGUNE, Basque Research and Technology Alliance (BRTA), Paseo de Miramón 182, 20014 Donostia-San Sebastian, Spain

e Ikerbasque, Basque Foundation for Science, 48013 Bilbao, Spain

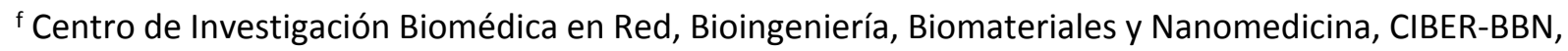
Paseo de Miramón 182, 20014 Donostia-San Sebastián, Spain

\begin{abstract}
Raman-encoded gold nanoparticles have been widely employed as photostable multifunctional probes for sensing, bioimaging, multiplex diagnostics, and surface-enhanced Raman scattering (SERS)-guided tumor therapy. We report a strategy toward obtaining a particularly large library of Au nanocapsules encoded with Raman codes defined by the combination of different thiol-free Raman reporters, encapsulated at defined molar ratios. The fabrication of SERS tags with tailored size and pre-defined codes is based on the in situ incorporation of Raman reporter molecules inside Au nanocapsules during their formation via Galvanic replacement coupled to seeded growth on Ag NPs. The hole-free closed shell structure of the nanocapsules is confirmed by electron tomography. The unusually wide encoding possibilities of the obtained SERS tags are investigated by means of either
\end{abstract}


wavenumber-based encoding or Raman frequency combined with signal intensity, leading to an outstanding performance as exemplified by 26 and 54 different codes, respectively. We additionally demonstrate that encoded nanocapsules can be readily bioconjugated with antibodies for applications such as SERS-based targeted cell imaging and phenotyping.

KEYWORDS: plasmonic nanocapsules, SERS tags, SERS library, combinatorial encapsulation, cell imaging

Gold nanoparticles (Au NPs) functionalized with Raman-active molecules have been extensively used as SERS tags for bioimaging applications, owing to their biocompatibility, simple surface chemistry and greater stability over Ag NPs. ${ }^{1-7} \mathrm{~A}$ wide range of SERS tags have been reported, based on Au NPs of different sizes and shapes encoded with various Raman reporters (RaRs) and conjugated to a wide range of biomolecules..$^{1-3,5,8-12}$ The main prerequisites of SERS tags for biosensing and imaging include high sensitivity, uniform and reproducible SERS response, targeting ability, biocompatibility, and long term stability. ${ }^{5,13-16}$

SERS tags can be readily fabricated by sequential functionalization of Au NPs with RaRs and specific antibodies. $5,11,14,17,18$ However, the complexity involved in their design and fabrication is considerably high, due to challenges related to achieving a high targeting efficiency while maintaining high SERS signals and reliability, as well as colloidal stability. Therefore, conventional SERS tags are often prepared by deposition of a protective coating layer (typically $\mathrm{SiO}_{2}$ or polymer) on RaR-encoded Au NPs, to prevent SERS tag aggregation and leaching of RaR molecules, ${ }^{19,20}$ as well as to facilitate the further functionalization with 
appropriate antibodies or other targeting moieties. ${ }^{8,11,17,21,22}$ On a different approach, SERS tags have been developed consisting of metallic core-shell structures with intrinsic hot spots at nanoscale gaps, where one or two RaRs can be embedded..$^{23-27}$ These nanostructures were proven to yield highly intense and stable SERS signals, but involve a rather complex fabrication process, and it is limited to thiolated RaRs.

Although one of the main arguments in favor of SERS tags, as compared to fluorescent dyes, is the narrowness of Raman peaks and the vast range of potentially available codes, codification is usually based on the adsorption of a limited number of thiolated molecules or dyes, either on the nanoparticle surface or at nanogaps, which in practice limits the encoding capabilities. An interesting alternative to extend the library of SERS tags relies on the combination of multiple RaRs. ${ }^{28}$ We hypothesized that such an expanded library could be achieved by entrapping RaRs in the hollow interior of gold nanoshells so that the whole outer surface would be available for bioconjugation. However, the synthesis of Au nanoshells is usually done by gold reduction on a colloidal template, such as silica or polystyrene spheres, ${ }^{29-}$ 32 thus leaving no hollow interior where RaRs could be effectively introduced. On the other hand, Au nanocages do have a hollow interior but the metal shells formed by galvanic replacement on sacrificial Ag NP templates are typically highly porous, so unable to retain molecular cargo inside..$^{3,4,33-35}$ As an alternative, we have recently shown that impervious $\mathrm{Au}$ nanoshells can be obtained by carrying out the Galvanic replacement reaction in the presence of a surfactant (hexadecyltrimethylammonium bromide, CTAB) and a mild co-reducing agent (ascorbic acid, AA). ${ }^{36}$ Complexation of CTAB with the gold salt precursor facilitates the reduction of $\mathrm{Au}^{3+}$ into $\mathrm{Au}^{+}$by $\mathrm{AA}$, significantly slowing down the reaction rate of Galvanic replacement while promoting the catalytic reduction of $\mathrm{Au}^{+}$into $\mathrm{Au}^{0}$ and closure of the shell. ${ }^{6,36}$ 
With this synthetic strategy in mind, we investigated the fabrication of SERS tags by trapping one or several RaRs inside hollow and closed Au nanoshells, during their formation from $\mathrm{Ag}$ nanospheres (below $100 \mathrm{~nm}$ ) via Galvanic replacement coupled to seeded growth (Scheme 1). This strategy would allow the combination of various RaRs in different molar ratios, thereby expanding the available library of SERS tags by means of both wavenumber-based encoding and wavenumber coupled to relative signal intensity. Thiol free RaRs were selected, to minimize interference in the fabrication process of Au nanocapsules, as well as to avoid blocking of their outer surface. Combination of multiple RaRs in the same nanoshells resulted in an exemplary library comprising up to 54 different codes. The closed shell morphology and the SERS enhancement were confirmed by electron tomography and Finite Difference Time Domain (FDTD) simulations, respectively. Additionally, we demonstrated the potential application of nanocapsule-based tags for SERS-based bioimaging through multiplex immunophenotyping of cancer cells.

\section{RESULTS AND DISCUSSION}

The overall synthetic procedure to obtain SERS tags through encapsulation of Raman-active dyes within hollow Au nanocapsules and covalent surface functionalization of biomolecules is depicted in Scheme 1. Simultaneous addition of tetrachloroauric acid $\left(\mathrm{HAuCl}_{4}\right), \mathrm{AA}$ and CTAB to a colloidal dispersion of Ag nanospheres mixed with one or several RaRs leads to a Galvanic replacement reaction, which is eventually coupled to seeded growth and dye encapsulation, resulting in dye-containing Ag-Au hollow nanoparticles (Scheme 1, step i). The fabrication of SERS tags concludes with the covalent bioconjugation of antibodies using thiolated PEG and EDC-NHS chemistry as shown in Scheme 1, step ii (see Experimental section for further details). 

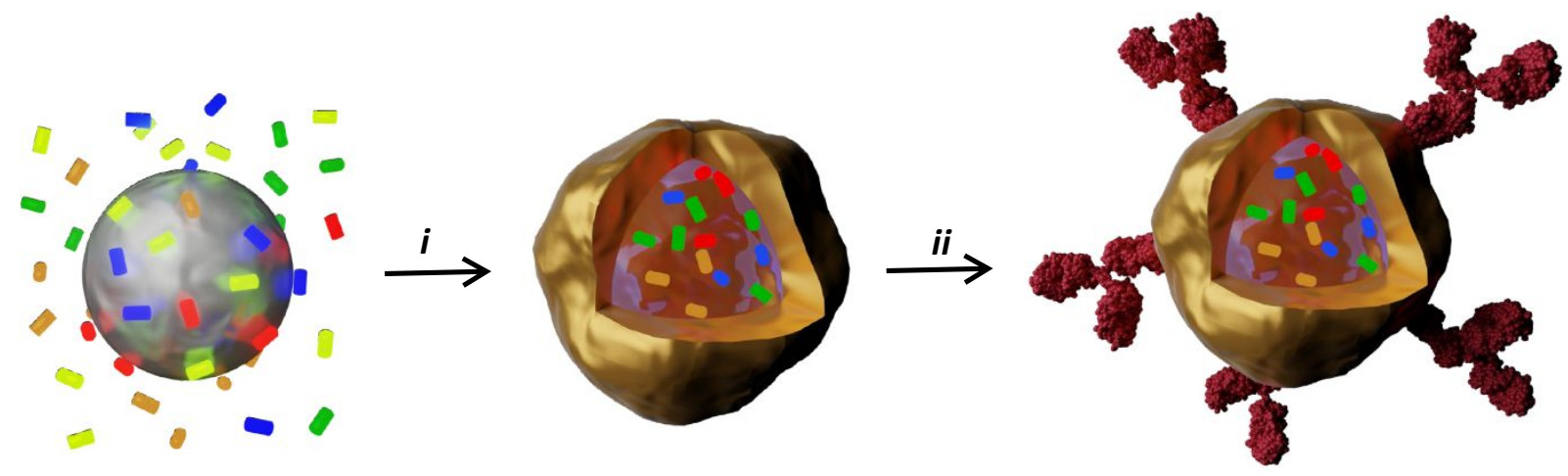

Scheme 1. Schematic representation of the synthesis of Raman reporter-encoded plasmonic nanocapsules through seed-mediated growth coupled to Galvanic replacement $(i)$ and subsequent bioconjugation (ii).

RaR molecules were specifically chosen to avoid thiol groups and thereby ensure uniform shell growth and RaR entrapment within the growing capsules. We monitored the formation of dyeencoded nanocapsules by UV-Vis-NIR spectroscopy. It should be noted that the high extinction coefficient of the metal particles does not allow us to observe any of the absorption features of the dyes. As shown in Figure $1 \mathrm{~A}$ for nanocapsules obtained from $58 \mathrm{~nm} \mathrm{Ag}$ nanoparticles (LSPR at ca. $440 \mathrm{~nm}$ ), controlled addition of Au salt gave rise to an initial decrease in extinction, together with a gradual red-shift and broadening of the localized surface plasmon (LSPR) band. At later reaction stages, when seeded growth became dominant, an increase of extinction intensity together with an LSPR blue shift was observed due to the gradual increase of Au shell thickness. The resulting nanocapsules presented a well-defined LSPR band centered around $600 \mathrm{~nm}$. The overall process was also investigated via the structural and compositional analysis of nanoparticles obtained for different $\mathrm{Ag}$ :Au ratios, by means of conventional 
transmission electron microscopy (TEM), high-angle annular dark-field scanning transmission electron microscopy (HAADF-STEM) and EDX mapping. Shown in Figure 1B-E are representative low-magnification TEM images of the sacrificial Ag nanoparticles and the particles obtained for increasing $\mathrm{Au} / \mathrm{Ag}$ ratio. The nanoparticles maintained a pseudospherical morphology, while increasing the overall size from $58 \pm 7 \mathrm{~nm}$ to $74 \pm 9 \mathrm{~nm}, 77 \pm 8 \mathrm{~nm}$ and 90 $\pm 10 \mathrm{~nm}$ when $\mathrm{Ag}: \mathrm{Au}$ molar ratios of $2.06,1.2$, and 0.76 were used, respectively (see Experimental section). Similar results were obtained in control experiments performed in the absence of Raman dyes, indicating that encapsulation of the dyes did not affect the formation of the nanocapsules. RaR-doped plasmonic capsules with different dimensions and optical features could be readily obtained by tuning the size of the sacrificial Ag templates. Results obtained using $\mathrm{Ag}$ nanoparticles with average sizes of $40 \mathrm{~nm}$ and $80 \mathrm{~nm}$ are shown in Figures S1 and S2.
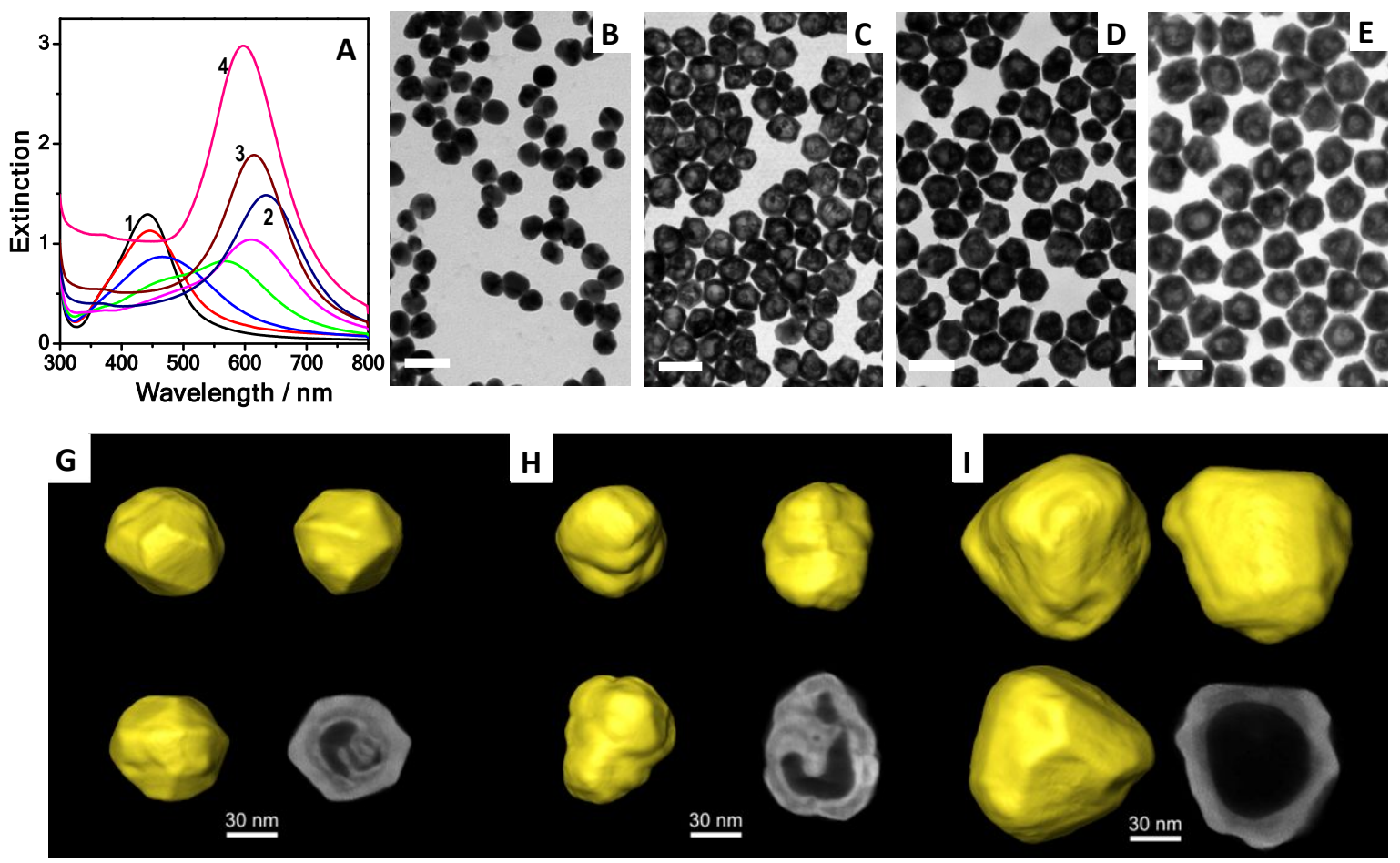

Figure 1. (A) UV-Vis-NIR spectra of silver nanoparticles before (1) and after Galvanic replacement with $\mathrm{Ag}$ :Au molar ratios ranging from 2.08 to 0.76 . (B-E) Representative TEM 
images of Ag nanoparticles (B, 1) and those obtained at different Ag:Au molar ratios; 2.06 (C, 2), $1.2(D, 3)$ and $0.76(E, 4)$. Scale bars represent $100 \mathrm{~nm}$. G-I. Visualizations of 3D electron tomography reconstructions of the particles obtained using Ag:Au molar ratios of 2.06 (G), 1.2 (H) and 0.76 (I). Three orthogonal views of an isosurface and one slice through the reconstruction are depicted for each particle.

We further investigated the potential presence of holes in the shell by electron tomography, under different synthesis conditions. ${ }^{37}$ Figure 1G-I depicts visualizations of 3D tomographic reconstructions from the analysis of representative nanocapsules obtained at $\mathrm{Ag}: \mathrm{Au}$ molar ratios of 2.06, 1.2 and 0.76 . The reconstructions indicate the presence of an inner void and a completely closed shell in all samples. At least 3 nanoparticles from each sample were investigated, to confirm the absence of holes in the shell. Animated visualizations of the obtained reconstructions are provided as Supplementary Information (Movies S1-S3). HAADFSTEM analysis (Figures 2A-C) also revealed the morphological transformation, with formation of a void surrounded by a whole shell, with increasing thickness at higher amounts of added Au salt. EDX elemental mapping showed that each nanocapsule was constituted by an inner Ag-rich shell and an outer Au-rich shell (see Figures $\mathbf{2}$ and S3). The EDX maps also confirmed the growth of the outer Au shell, as more gold salt precursor was added. Thus, the average Au content in the particles increased from $50 \%$ to $85 \%$, as the $\mathrm{Ag}$ :Au molar ratio decreased from 2.06 to 0.76 (Figure 2D). It should be noted that similar morphological transformations were observed when using $\mathrm{Ag}$ seeds with diameters of $40 \mathrm{~nm}$ and $80 \mathrm{~nm}$ (Figure S3). Scanning electron microscopy images additionally evidenced the absence of pinholes on the Au shells (Figure S4 in the SI). We can thus conclude that this synthesis method can be generally used to obtain completely closed nanocapsules with tunable dimensions. 

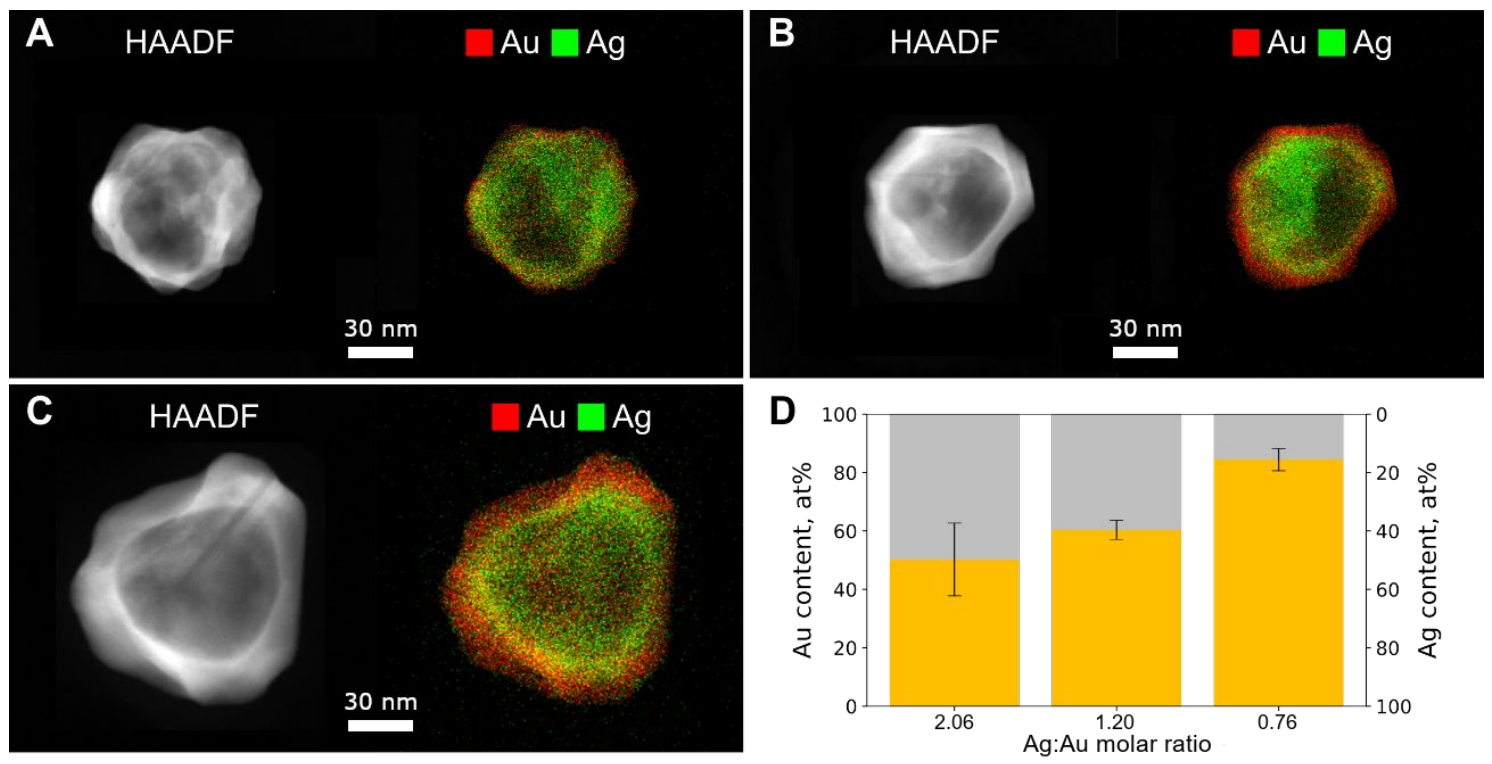

Figure 2. (A-C) Representative HAADF-STEM images and EDX maps of nanoparticles obtained for different Ag:Au molar ratios: 2.06 (A), 1.2 (B) and 0.76 (C). (D) Relative content of $\mathrm{Au}$ (yellow) and Ag (grey) in nanoparticles of samples at different Ag:Au molar ratios, as labeled. Error bars correspond to the standard deviation between measurements for 5 different nanoparticles.

The performance of SERS tags is largely determined by the intensity and distribution of the electromagnetic field upon laser excitation. SERS enhancement is often estimated by averaging $\left|E / E_{0}\right|^{4}$ over the amount of Raman reporters, where $E$ and $E_{0}$ are the local and incident electromagnetic fields, respectively. We performed FDTD modeling to simulate the LSPR, as well as the enhanced electromagnetic field (intensity and distribution) around the nanocapsules. We selected a model for a spherical nanocapsule dispersed in water, with an internal void filled with water and a complete shell made of a thin inner Ag layer $(2.5 \mathrm{~nm})$ and a thicker outer Au layer (Figure 3A). Simulations were carried out for nanocapsules with external diameters of 63,89 , and $119 \mathrm{~nm}$ and internal voids of 33,54 , and $75 \mathrm{~nm}$, respectively, as estimated from TEM and STEM analysis, for nanocapsules obtained from Ag seeds of ca. 40, 60 and $80 \mathrm{~nm}$. As shown in Figure S5, the calculated extinction spectra are in good 
agreement with the experimental data. The electric field enhancement was simulated at three linearly polarized excitation laser lines, namely 532, 633 and $785 \mathrm{~nm}$. The results show that, regardless of the nanocapsules dimension and the laser line, the dipolar electric field exhibited a local maximum at the external water-gold interface (Figures 3B and S6). However, an almost homogeneous electric field distribution was also observed in the whole interior volume of the nanocapsule, as clearly evidenced in Figure 3E,D through electric field profiles parallel and perpendicular to the polarization of the incident light. Although (for both polarizations) the electric field generated at the outer gold-water interface decays exponentially, an almost constant field enhancement is present in the whole volume of the nanocapsule void.

Calculation of the total Raman enhancement for the three analyzed nanocapsules at three excitation laser lines was performed, considering a constant electric field distribution inside the void and integrating the Raman enhancement factor over the whole volume. The results are summarized in Figure S7, showing that the nanocapsule with $89 \mathrm{~nm}$ diameter and internal void of $54 \mathrm{~nm}$ displays the highest total Raman enhancement upon excitation at $633 \mathrm{~nm}$. We, therefore, used nanocapsules with $90 \mathrm{~nm}$ in diameter and $54 \mathrm{~nm}$ of internal void (prepared from ca. $60 \mathrm{~nm} \mathrm{Ag} \mathrm{seeds)} \mathrm{throughout} \mathrm{all} \mathrm{encoding} \mathrm{and} \mathrm{imaging} \mathrm{experiments.}$ 

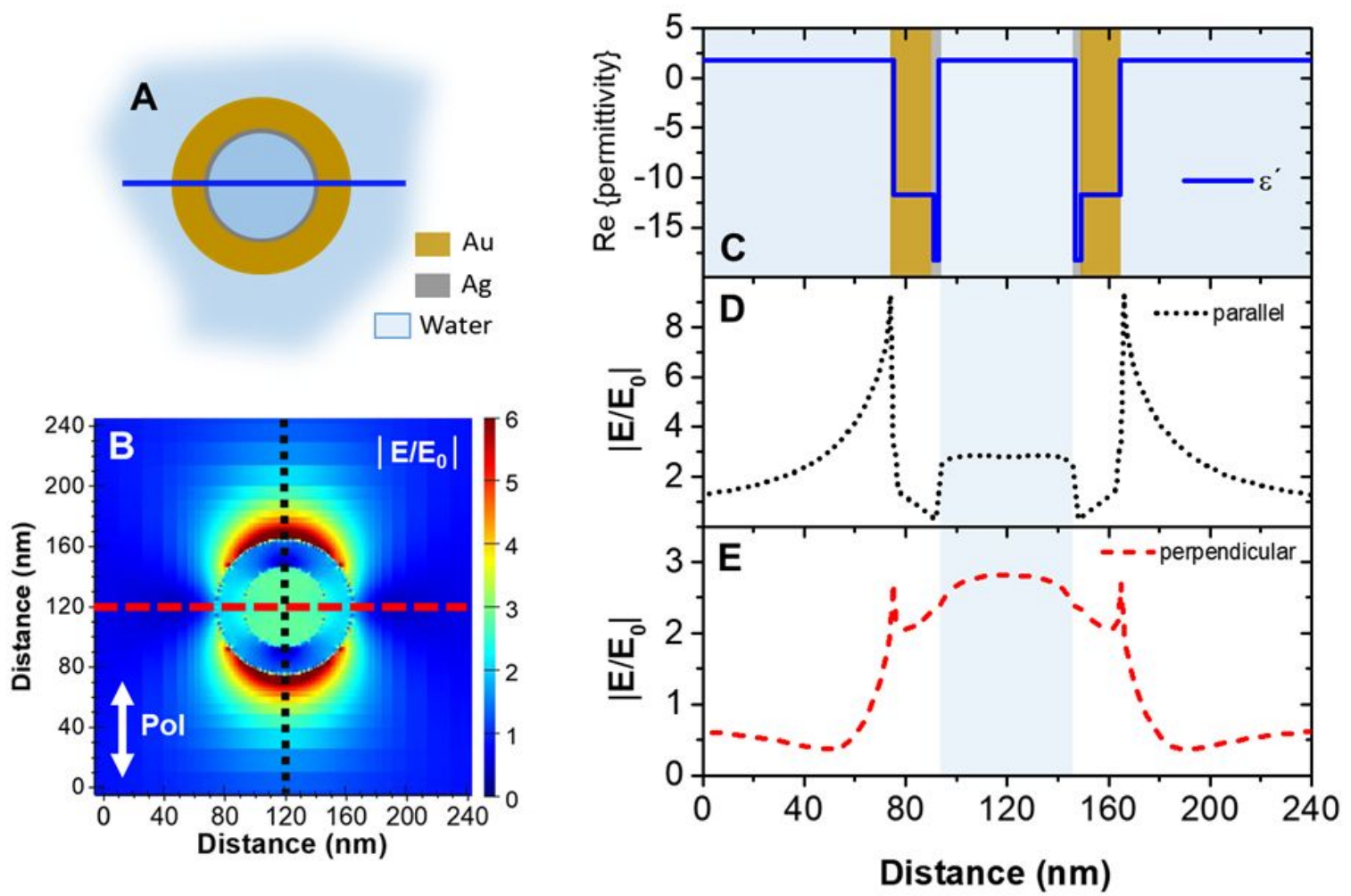

Figure 3. (A) Model of a hollow nanocapsule used for parametrization, indicating the material properties from the interior to the surface (water-blue, silver-grey, gold-brown). (B) Plot of the calculated electric field distribution for a nanocapsule with $89 \mathrm{~nm}$ outer diameter, $54 \mathrm{~nm}$ internal void and $2.5 \mathrm{~nm}$ inner Ag layer. The incident light (633 nm) was linearly polarized in the vertical direction. (C) Profile of the real part of the permittivity along the blue line in (A). (D-E) Electric field enhancement profiles parallel (D, black dotted line in B) and perpendicular $(E$, red dashed line in $B$ ) to the polarization of the incident light (black dotted line in (B)). Blue areas in (D) and (E) indicate the inner void.

To test our hypothesis, we prepared SERS tags containing five different Raman reporters with no specific functional groups having affinity toward the metal surface. Specifically, the selected dyes were: malachite green (MG), Nile blue (NB), methylene blue $(\mathrm{MB})$, Astra blue $(\mathrm{AB})$ and crystal violet $(\mathrm{CV})$, and their molecular structures are shown in Figure 4. The SERS spectra of all five SERS-encoded nanoparticles were recorded under excitation with $633 \mathrm{~nm}$ (Figure 4B) and 532 and $785 \mathrm{~nm}$ laser lines (Figure S8), invariably displaying the characteristic SERS peaks from the corresponding RaR. The efficiency of the 
different SERS-encoded nanoparticles was tested in aqueous dispersion, at $633 \mathrm{~nm}$ excitation, by plotting the SERS intensity as a function of particle concentration (Figure S9). All plots show a linear correlation, with small standard deviations over the concentration range between

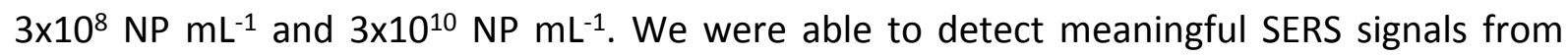
encoded nanoparticles, down to concentrations as low as $10^{8}-10^{9} \mathrm{NP} \mathrm{mL}^{-1}$, i.e. values similar to previously reported SERS tags. ${ }^{11,38,39}$ The stability of the SERS tags was investigated by analyzing the time evolution of their extinction and SERS spectra. The results clearly showed that our SERS tags featured long-term stability, as reflected by constant SERS and LSPR signals, for at least 3 months (results for $A B$ and MG are provided in Figure S10).

To unambiguously demonstrate the encapsulation of the RaR molecules inside nanoshells and discard their adsorption onto the outer surface, a control experiment was performed as follows. Nanocapsules were prepared in the presence of $A B$ and subsequently, MG was added, so it can adsorb on the outer surface. After surface modification with thiolated polyethylene glycol (the zeta potential changed from $+48.7 \pm 0.8 \mathrm{mV}$ to $-20.3 \pm 1.2 \mathrm{mV}$ ), SERS tags were transferred into ethanol and washed by several centrifugation/redispersion cycles to remove non-encapsulated molecules. SERS analysis of the colloidal dispersions consistently revealed characteristic Raman peaks for $A B$, confirming efficient encapsulation, but additional peaks from MG were only recorded after addition on preformed capsules and disappeared after washing, thereby confirming removal of MG only (Figure S11). We also confirmed that SERS tags prepared in the presence of increasing RaR concentrations displayed increased SERS signal intensities (Figure S12), again indicating efficient molecular trapping inside the nanocapsules. 
Arguably, the main advantage of this method is the possibility to trap two or more Raman reporters simultaneously within each nanocapsule, potentially leading to enhanced multiplexing. Therefore, a binary code for each Raman reporter according to its presence or its absence in the label was applied to each of the five selected RaRs ( $M G, C V, N B, A B, M B$ ). All possible combinations would thus result in 31 different codes (see Section 4 in the SI for further details). Figure S13 shows representative SERS spectra for 26 out of the 31 possible combinations. To differentiate all the codes, a multivariate analysis based on principal component analysis (PCA) was performed using representative SERS spectra. The remaining 5 codes were not included as they were not easily distinguishable in the PCA. The 3D PCA score plot showed that all Raman-encoded nanocapules can be clearly identified by the first three principal components (PC1, PC2, and PC3, which account for $43.3 \%, 40.5 \%$ and $8.2 \%$ of the total explained variance, respectively. See Figures 4C and 4D). Additionally, the PCA loadings plot from the first, second and third principal components (see Figure S14 in the SI) show the main vibrational bands that are to be considered to differentiate the different mixtures in PC1, PC2 and PC3, respectively. 
A

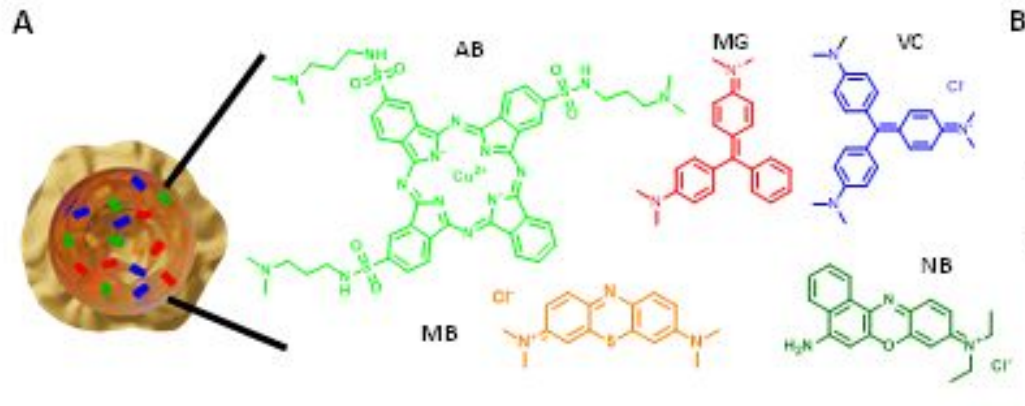

c

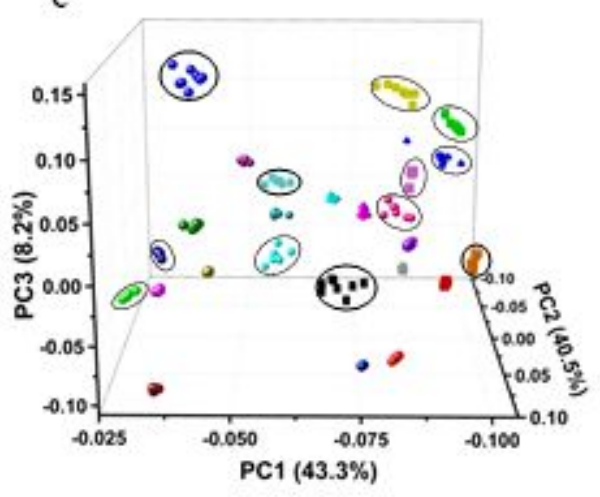

D

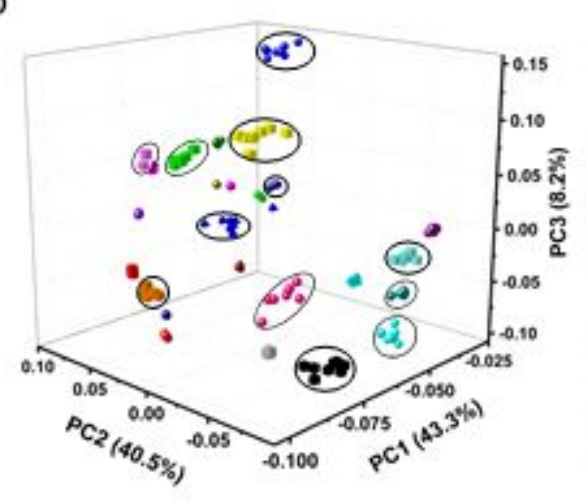

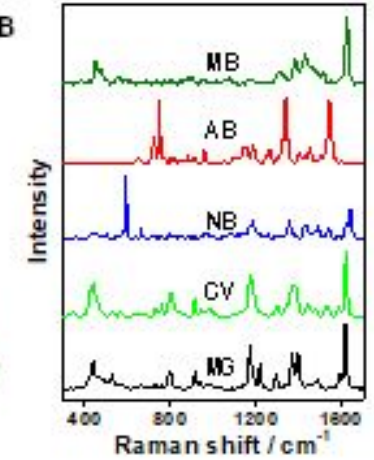

Figure 4. (A) Schematic representation of a plasmonic nanocapsule encoded with five different Raman reporters: Malachite Green (MG), Crystal Violet (CV), Nile Blue (NB), Astra Blue (AB) and Methylene Blue (MB). (B) Representative SERS spectra of capsules encoded with each of the five RaRs. (C, D) Two different views of a 3D PCA score plot for the first three PCs from 26 (out of 31) SERS tags obtained by combination of five different Raman reporters. The ellipses in $C$ and $D$ are drawn on the same mixtures to facilitate their identification.

We subsequently investigated an extension of the encoding possibilities through encryption based on combinations of Raman frequency with SERS intensity. We thus prepared SERS tags containing two different dyes, $A B$ and $M G$, in different molar ratios. Figure $5 A$ shows the characteristic SERS spectra of the resulting plasmonic capsules with different $A B$ and MG ratios. Analysis of the relative SERS signal intensity between one peak characteristic of $A B$ $\left(1539 \mathrm{~cm}^{-1}\right)$ and another of MG $\left(1614 \mathrm{~cm}^{-1}\right)$ (square areas in Figure $\left.5 \mathrm{C}\right)$ indicates the possibility of generating a 2D barcode library using a single excitation laser line. By normalizing the 
spectra to the highest peak intensity and identifying up to ten levels for each reporter, would generate up to 20 different codes. Figure 5 shows representative SERS spectra of 17 different $A B: M G$ ratios and their corresponding barcodes. A similar approach could be applied to a combination of three dyes $(A B, M G$ and $N B)$ since each of them presents at least one peak that is not present in the others $\left(1539 \mathrm{~cm}^{-1}, 1614 \mathrm{~cm}^{-1}\right.$ and $591 \mathrm{~cm}^{-1}$, respectively, see Figure S15). Thus, by normalizing to the highest peak and identifying up to ten levels for each RaR, it is possible to generate up to 300 different codes. Figure $\mathbf{S 1 5}$ shows 37 representative codes (out of 300). Combination of five different Raman reporters would make it possible to encode up to 49,995 different tags (see section 4 in the SI, for a detailed description of the encoding possibilities).
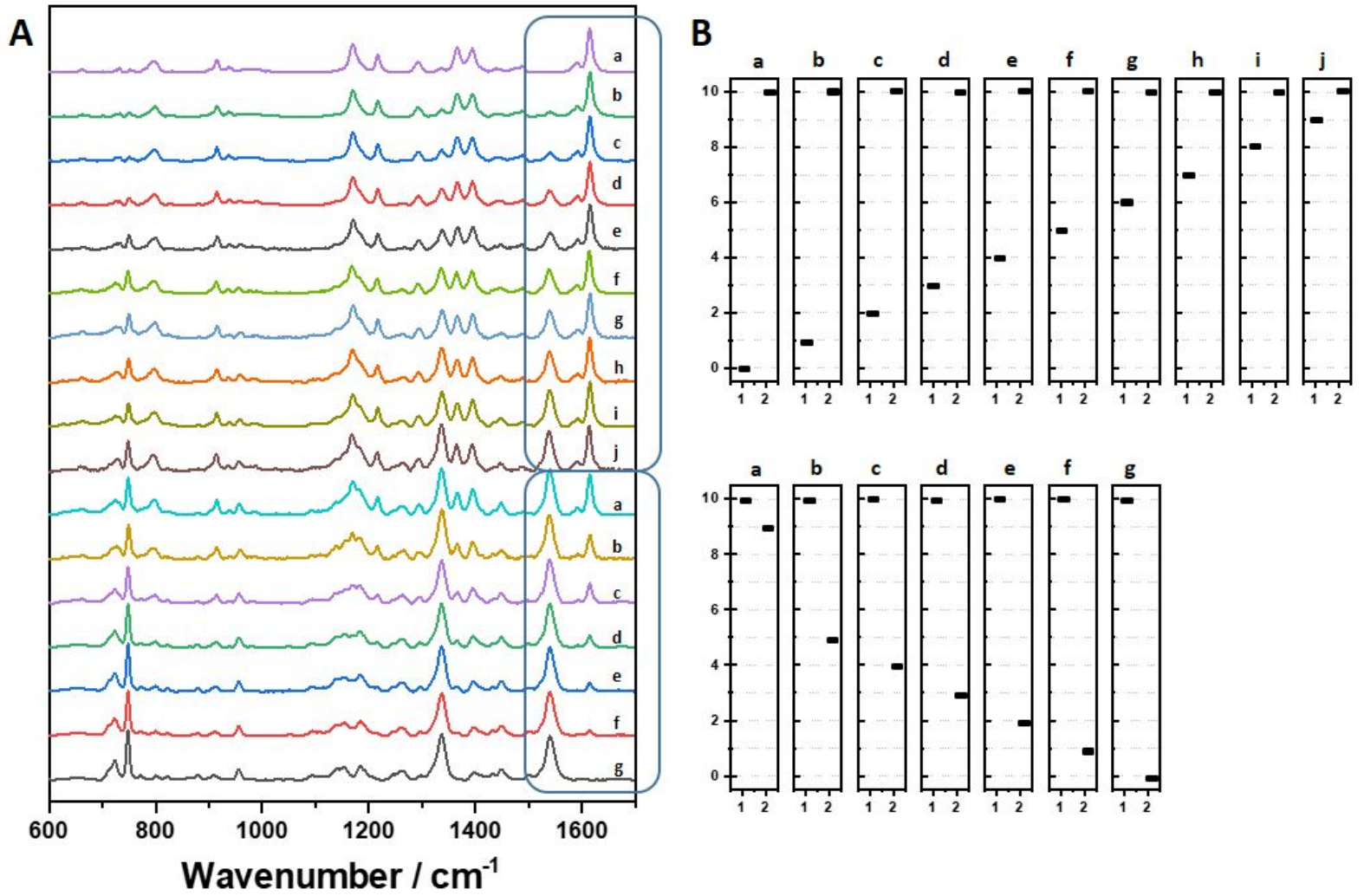

Figure 5. (A) Representative SERS spectra obtained by mixing $A B$ and $M G$ at different molar ratios. The square regions highlight the characteristic SERS peaks of both dyes, normalizing 
the spectra to the highest peak intensity. (B) 2D spectral bar code recognizing up to ten levels for each reporter.

We further proceeded with antibody $(\mathrm{Ab})$ conjugation and analyzed the potential interference of Abs and other biomolecules, on the SERS tags signal. Therefore, nanocapsules were first encoded with $M G, N B$ or $A B$, and bioconjugated with antibodies against three different cell surface receptors, namely epidermal growth factor receptor (EGFR), epithelial cell adhesion molecule (EpCAM), and homing cell adhesion molecule (CD44), respectively. After incubation with a human epithelial carcinoma A431 cell line expressing EGFR, EpCAM, and CD44, SERS spectra were recorded for the different samples. Figure S16 shows SERS spectra for the different SERS tags, before and after bioconjugation and incubation. It should be noted that no additional SERS signals were identified in the spectra, clearly showing interference-free detection in complex biological samples.

To further investigate the reliability and bioimaging capabilities of our SERS tags, we carried out simultaneous SERS detection of EGFR, EPCAM, and CD44 expressed in A431 cells and nontumoral murine fibroblast 3 T3 2.2 cells. Whereas the A431 cell line expresses all three surface receptors, the 3 T3 2.2 cell line only expresses CD44. ${ }^{11}$ Our SERS tags demonstrated outstanding selectivity and specificity when incubated with A431 and 3T3 2.2 cell lines separately (Figures S17-S19). Further control experiments combining SERS analysis and immunostaining were also performed for the detection of EGFR and CD44 in a co-culture of A431 and 3T3 2.2 cells (Figure S20). Finally, multiplex detection of the three surface receptors was demonstrated in the cell co-culture, as exemplified the SERS analysis shown in Figures S21 and S22 for two groups of cells. SERS mappings were recorded using the peaks at 1617 $\mathrm{cm}^{-1}, 1640 \mathrm{~cm}^{-1}$, and $1537 \mathrm{~cm}^{-1}$ to evaluate the spatial distribution of MG (EGFR receptor), NB 
(CD44) and $A B$ (EpCAM), respectively. The results clearly demonstrate differentiation of tumor A431 cells (expressing EGFR, CD44 and EpCAM) from non-tumor 3T3 2.2 cells (expressing CD44 only), as well as the reliability of the assay and the multiplexing capabilities of SERS tags based on hollow nanocapsules.

\section{CONCLUSIONS}

In summary, SERS tags with outstanding encoding capabilities were prepared by in situ encapsulation/trapping of non-thiolated RaR molecules inside plasmonic nanocapsules. The proposed system allows the use of a wider range of RaRs and their mixtures, and largely expands the encoding possibilities of spectral encryption through both wavenumber-based encoding and wavenumber coupled to signal intensity encoding. We demonstrated that encoded nanocapsules offer an ideal platform for SERS-based targeted multiplexing of cellular membrane receptors. The concept presented in this work is versatile and expected contribute to the incorporation of various biomolecules for biomedical applications such as flowcytometry, enzyme-linked immunosorbent assay (ELISA) or automated microscopy.

\section{EXPERIMENTAL SECTION}

Chemicals. Silver nitrate $\left(\mathrm{AgNO}_{3}, \geq 99 \%\right)$, sodium citrate tribasic dihydrate ( $\left.\geq 98 \%\right)$, tannic acid,

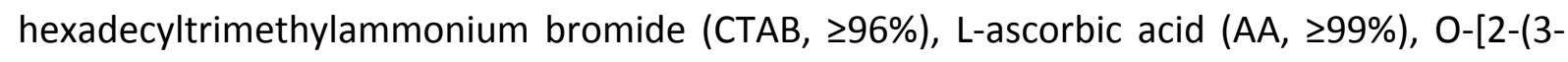
mercaptopropionylamino)ethyl]-O'-methylpolyethylene glycol (PEG-SH, MW 5,000), poly(ethylene glycol) 2-mercaptoethyl ether acetic acid (HS-PEG-COOH, MW 5,000), malachite green oxalate salt (MG), Nile blue chloride (NB), methylene blue (MB), methanol ( $\geq 99.9 \%)$, polyethylene glycol sorbitan monolaurate (Tween 20), N-hydroxysuccinimide sodium salt (NHS, $\geq 97.0 \%), \mathrm{N}$-(3-dimethylaminopropyl)-N'-ethylcarbodiimide hydrochloride (EDC), bovine serum albumin (BSA, $\geq 98 \%$ ), fetal bovine serum (FBS), EDTA $10 \mathrm{mM}$ saline solution, formalin solution and phosphate buffer saline (PBS) were purchased from Sigma-Aldrich. Hydrogen tetrachloroaurate(III) trihydrate $\left(\mathrm{HAuCl}_{4} \cdot 3 \mathrm{H}_{2} \mathrm{O}\right)$ and 2-(N-morpholino)ethanesulfonic acid (MES) monohydrate (98\%) were supplied by Alfa Aesar and Dulbecco's modified Eagle's 
medium (DMEM), penicillin streptomycin solution and Prolong antifade reagent were supplied by ThermoFisher Scientific. Astra blue (AB) and crystal violet (CV, 90\%) were obtained from Marker Gene Technologies and Analema, respectively. All chemicals were used as received. Pure grade ethanol and Milli-Q grade water were used as solvents.

Regarding the antibodies, rabbit anti-EpCAM (ab225894), rat anti-CD44 (ab119348), mouse anti-EGFR (ab30), goat anti-rat Alexa 647 (ab150167), goat anti-rabbit Alexa 488 (ab150077) were acquired from Abcam, goat anti-mouse CF488 (SAB4600388) from Sigma.

Synthesis of silver nanoparticles. Citrate-stabilized Ag NPs with different sizes were prepared by the seeded growth method reported by Bastús et al. ${ }^{40} 100 \mathrm{~mL}$ of an aqueous solution containing $5 \mathrm{mM}$ sodium citrate and $0.1 \mathrm{mM}$ tannic acid in a three-neck round bottom flask was heated up to boiling and immediately after $1 \mathrm{~mL}$ of $25 \mathrm{mM}$ silver nitrate was added under vigorous stirring. The solution color changed indicating the formation of seeds. After 15 minutes, $19.5 \mathrm{~mL}$ of the resulting seeds was extracted and $16.5 \mathrm{~mL}$ of Milli-Q water was added. After setting the temperature of the solution to $85^{\circ} \mathrm{C}, 0.5 \mathrm{~mL}$ of $100 \mathrm{mM}$ sodium citrate, 1.5 $\mathrm{mL}$ of $2.5 \mathrm{mM}$ tannic acid and $1 \mathrm{~mL}$ of $25 \mathrm{mM}$ silver nitrate were sequentially added. After 30 minutes this solution was used as a new seed solution and the process was repeated up to obtain the desired size. Thus, Ag nanoparticles of ca. 40, 60 and $80 \mathrm{~nm}$ were obtained. Finally, Ag NPs were centrifuged at 18030g, 11180g or 9050g (for 40, 60 or $80 \mathrm{~nm} \mathrm{Ag} \mathrm{NPs,} \mathrm{respectively)}$ for $5 \mathrm{~min}$ and the pellet redispersed in the same amount of water. The concentration in terms of Ag metal was $0.76,1.03$ and $1.18 \mathrm{mM}$ for $\mathrm{Ag}$ colloids of 40,60 or $80 \mathrm{~nm}$, respectively.

Synthesis of SERS tags. The synthesis was performed as previously reported with modifications. ${ }^{36}$ Briefly, $2 \mathrm{~mL}$ of Ag colloid $(0.76,1.03$ and $1.18 \mathrm{mM}$ for Ag NPs of 40,60 or 80 $\mathrm{nm}$, respectively) was added to $8 \mathrm{~mL}$ of $0.1 \mathrm{M} \mathrm{CTAB}$ in water, followed by the addition of 0.2 $\mathrm{mL}$ of $0.2 \mathrm{M} \mathrm{AA}$ in water and $150 \mu \mathrm{L}$ of a stock solution containing a Raman reporter $(1 \mathrm{mM}$ for $A B, N B$ and $M B$ (in ethanol), $10 \mathrm{mM}$ for $M G$ (in water) and $24 \mathrm{mM}$ for $\mathrm{CV}$ (in water)). Finally, $0.5 \mathrm{mM} \mathrm{HAuCl}_{4}$ was gradually added under continuous stirring at a constant rate of $50 \mu \mathrm{L} / \mathrm{min}$ using a syringe pump. For the time-resolved UV-Vis-NIR characterization and TEM analysis, different aliquots were extracted at the desired times (Ag:Au molar ratios) and the particles were washed by centrifugation to remove the excess of dye and redispersed in the same volume. Finally, encoded nanocapsules were purified twice by centrifugation at $3420 \mathrm{~g}$ (20 $\mathrm{min}$ ) and $2790 \mathrm{~g}(5 \mathrm{~min}$ ) and redispersed in $1 \mathrm{~mL}$ of CTAB $1 \mathrm{mM}$. After this step, the encoded 
nanocapsules show a $\zeta$-potential of $+48.4 \pm 1.1 \mathrm{mV}$. Non-encoded hollow nanocapsules were fabricated similarly but without adding the dye.

Bioconjugation of encoded hollow nanocapsules was performed by diluting $125 \mu \mathrm{L}$ of the nanocapsules to $1 \mathrm{~mL}$ and subsequently adding HS-PEG-COOH $(115 \mu \mathrm{L} 3.9 \mathrm{mg} / \mathrm{mL}$, ca. 100 molecules $/ \mathrm{nm}^{2}$ ) under vigorous stirring. After keeping the mixture undisturbed for 3 hours, the colloids were washed twice by centrifugation (690 g for $15 \mathrm{~min}$ ) and redispersion in $1 \mathrm{~mL}$ of $50 \mathrm{mM}$ MES buffer ( $\mathrm{pH}=6,0.05 \%$ Tween 20). After functionalization with HS-PEG-COOH, the nanocapsules showed a $\zeta$-potential of $-25.3 \pm 1.6 \mathrm{mV}$. After the second wash, the supernatant was removed and $100 \mu \mathrm{L}$ of a $10 \mathrm{mM}$ MES buffer $(\mathrm{pH}=6)$ containing $15 \mathrm{mg}$ of EDC and $18 \mathrm{mg}$ of NHS was added under stirring. After $30 \mathrm{~min}$ under stirring, the colloidal dispersion was diluted by adding $1.5 \mathrm{~mL}$ of PBS $1 \mathrm{X}$ (0.05\% Tween 20) and centrifuged twice (690g for $8 \mathrm{~min}$ ). After the second washing, the antibody conjugation reaction was performed by adding $10 \mu \mathrm{L}$ of antibody $(1 \mathrm{mg} / \mathrm{mL})$ for $1 \mathrm{~h}$ under $30 \mathrm{rpm}$ rotary motion followed by overnight incubation at $4{ }^{\circ} \mathrm{C}$. Next, the samples were diluted with $1.5 \mathrm{~mL}$ of PBS $1 \mathrm{X}(0.05 \%$ Tween 20), washed twice $(690 \mathrm{~g} 5 \mathrm{~min})$ by centrifugation. Finally, the SERS tags were redispersed in $400 \mu \mathrm{L}$ of PBS $1 \mathrm{X}$ containing $1 \%$ of BSA. The SERS tags were stored until use at $4{ }^{\circ} \mathrm{C}$. After bioconjugation, the nanocapsules showed a $\zeta$-potential of $c a$. - $19 \mathrm{mV}$.

Cell Lines and In Vitro Culture Conditions. The human epidermoid carcinoma cell line A431 (ATCC, CRL-1555) and NIH 3T3 clone 2.2 (3T3 2.2) of murine fibroblasts, were grown as a monolayer in DMEM, supplemented with $10 \% \mathrm{FBS}$, penicillin $\left(100 \mathrm{U} \mathrm{mL}^{-1}\right)$, and streptomycin $\left(100 \mu \mathrm{g} \mathrm{m} \mathrm{m}^{-1}\right)$ at $37^{\circ} \mathrm{C}$ in a humidified atmosphere containing $5 \% \mathrm{CO}_{2}$.

Adhesion of SERS nanocapsules to in vitro cultured cells. A431 and 3T3 2.2 cells $\left(2 \times 10^{6}\right)$ were detached from the flask with $10 \mathrm{mM}$ EDTA in PBS and then rinsed twice with PBS by centrifugation ( $34 \mathrm{~g}$ for $4 \mathrm{~min})$. The cell lines in PBS $(400 \mu \mathrm{L})$ were then incubated either separately, or in a 1:1 mixture, with $100 \mu \mathrm{L}$ of as-prepared SERS tags for $1 \mathrm{~h}$ at room temperature in an orbital shaker. Next, the cells were washed three times by centrifugation with $5 \mathrm{~mL}$ of PBS to remove unbound SERS tags. The cells were then fixed with $30 \mu \mathrm{L}$ of formalin. For Raman analysis, $5 \mu \mathrm{L}$ of the cell suspension was combined with $2 \mu \mathrm{L}$ of Prolong and mounted on glass slides. 
Immunofluorescence staining, Raman and fluorescence microscopy analyses. The procedure was carried out as described previously. ${ }^{11}$ Before seeding the cells, two perpendicular lines were marked at the center of a coverslip with a glasscutter to allow localization of a given group of cells under both microscopes. A 1:1 mixture of A431 and 3T3 2.2 cells were seeded on sterile coverslips (13 mm diameter, VWR international), placed in a 24-well plate at a density of 15000 cells/well and grown for $18 \mathrm{~h}$. The cells were washed three times with $1 \mathrm{~mL}$ of PBS at room temperature, fixed with $0.5 \mathrm{~mL}$ of formalin for $20 \mathrm{~min}$ at room temperature, and washed three times with $1 \mathrm{~mL}$ of PBS. The coverslips were transferred to a wet chamber and blocked with $60 \mu \mathrm{L}$ of $10 \%(\mathrm{v} / \mathrm{v})$ goat serum solution in PBS for $1 \mathrm{~h}$. The coverslips were washed by immersion five times in PBS $(100 \mathrm{~mL})$, placed again in the wet chamber, and incubated with $60 \mu \mathrm{L}$ of $10 \%(\mathrm{v} / \mathrm{v})$ goat serum solution in PBS containing mouse anti-EGFR antibodies (1:100) for $1 \mathrm{~h}$ at room temperature. Subsequently, the coverslips were washed by immersion ten times in PBS. Next, the coverslips were incubated for $30 \mathrm{~min}$ at room temperature with $60 \mu \mathrm{L}$ of a $10 \%(\mathrm{v} / \mathrm{v})$ goat serum solution in PBS containing goat anti-mouse CF488 secondary antibodies (1:500). The samples were washed in PBS as indicated above, mounted with $2 \mu \mathrm{L}$ of Prolong on glass slides. The cells located at the intersection of the lines marked on the glass coverslips were analyzed under the Raman microscope and subsequently by fluorescence microscopy (Nikon NiE).

Characterization methods. UV-visible-NIR absorption spectra were recorded using Agilent 8453 or Cary 5000 spectrophotometers. Transmission electron microscopy (TEM) analysis was performed in a JEOL JEM 1010 microscope operating at an acceleration voltage of $100 \mathrm{kV}$. High-angular annular dark-field scanning TEM (HAADF-STEM) imaging, electron tomography and energy-dispersive X-ray spectroscopy (EDXS) were performed using a FEI Tecnai Osiris electron microscope equipped with a Super-X detector operated at $200 \mathrm{kV}$. Tomographic series were acquired between $+75^{\circ}$ and $-75^{\circ}$ sample tilt angles with a $3^{\circ}$ tilt step, using a Fischione Model 2020 tomography sample holder. After aligning the images in the tilt series by a cross-correlation based algorithm, 3D reconstructions were obtained using the expectation maximization (EM) method as implemented in ASTRA Toolbox. ${ }^{41}$ Z-Potential was determined through electrophoretic mobility measurements using a Zetasizer Nano $\mathrm{S}$ (Malvern Instruments, Malvern UK). 
Raman and SERS measurements were conducted with a Renishaw InVia Reflex system. The spectrograph used a high resolution grating (1200 or 1800 grooves $\mathrm{cm}^{-1}$ ) with additional bandpass filter optics, a confocal microscope and a 2D-CCD camera. SERS characterization was done using a macro- sampler accessory to measure in the liquid state. Laser excitation was carried out at 532, 633 and $785 \mathrm{~nm}$ with $31.1,7.77$ or $68.3 \mathrm{~mW}$ of maximum power, respectively, and $10 \mathrm{~s}$ acquisition time. SERS mappings were recorded using the SERS pointmapping method with a 20X objective (N.A. 0.40), which provided a special resolution of 3.0 $\mu \mathrm{m}^{2}$ (y-axis step). It created a spectral image by measuring the SERS spectrum of each pixel of the image, one at a time. A computer-controlled $x-y$ translation stage was scanned in $3.0 \mu \mathrm{m}$ (x-axis) and $3.0 \mu \mathrm{m}$ (y-axis) steps. The SERS images of each well were decoded using the characteristic peak intensities of the three Raman reporter molecules using WiRE software $\mathrm{V}$ 3.4 (Renishaw, UK). SERS spectra were analyzed using Grams software (Thermo Scientific, USA).

FDTD simulations. Finite Difference Time Domain (FDTD) simulations of nanocapsules were carried out using the commercial software Lumerical Solutions, Inc. Perfectly Matched Layers (PML) were used as boundary conditions of the simulation area, with a Total-Field ScatteredField (TFSF) linearly polarized light as source. Optical power box monitors were selected to estimate absorption and scattering cross-sections, while Frequency Domain Field Profile Monitors were used to obtain electromagnetic field spatial distributions.

\section{ASSOCIATED CONTENT}

\section{Supporting Information}

The Supporting Information is available free of charge at https://pubs.acs.org/

Additional nanocapsule characterization, simulations of extinction spectra and electric field distribution and SERS characterization.

\section{AUTHOR INFORMATION}

\section{Corresponding Authors}

Jorge Pérez-Juste - CINBIO, Universidade de Vigo, Departamento de Química Física, Campus Universitario As Lagoas, Marcosende, 36310 Vigo, Spain; orcid.org/0000-0002-4614-1699; Email: juste@uvigo.es 
Isabel Pastoriza-Santos - CINBIO, Universidade de Vigo, Departamento de Química Física, Campus Universitario As Lagoas, Marcosende, 36310 Vigo, Spain; orcid.org/0000-0002-1091-1364; Email: pastoriza@uvigo.es

\section{Notes}

The authors declare no competing financial interest.

\section{ACKNOWLEDGMENTS}

L.M.L.-M. acknowledges financial support from the European Research Council (ERC-AdG4DbioSERS-787510) and the Spanish State Research Agency (Grant No. MDM-2017-0720 and PID2019-108954RB-I00). I.P.-S. and J.P.-J. acknowledge financial support from the Spanish State Research Agency (Grant No. MAT2016-77809-R)) and Ramon Areces Foundation (Grant No. SERSforSAFETY). G.B. acknowledges financial support from CINBIO (Grant number ED431G 2019/07 Xunta de Galicia). S.B. and A.S. acknowledge financial support by the Research Foundation Flanders (FWO grant G038116N). This project received funding as well from the European Union's Horizon 2020 research and innovation program under grant agreement No 731019 (EUSMI). S.B. acknowledges support from the European Research Council (ERC Consolidator Grant \#815128 REALNANO). We thank Carlos Fernández-Lodeiro and Daniel García-Lojo for their helpful contribution to the SEM characterization and SERS analysis and Veronica Montes-García for her fruitful contribution in the PCA analysis.

\section{REFERENCES}

(1) Lane, L. A.; Qian, X.; Nie, S. SERS Nanoparticles in Medicine: From Label-Free Detection to Spectroscopic Tagging. Chem. Rev. 2015, 115, 10489-10529.

(2) Guerrero-Martínez, A.; Barbosa, S.; Pastoriza-Santos, I.; Liz-Marzán, L. M. Nanostars Shine Bright for You: Colloidal Synthesis, Properties and Applications of Branched Metallic Nanoparticles. Curr. Opin. Colloid Interface Sci. 2011, 16, 118-127.

(3) Goris, B.; Polavarapu, L.; Bals, S.; Van Tendeloo, G.; Liz-Marzán, L. M. Monitoring Galvanic Replacement through Three-Dimensional Morphological and Chemical Mapping. Nano Lett. 2014, 14, 3220-3226.

(4) Sun, X.; Kim, J.; Gilroy, K. D.; Liu, J.; König, T. A. F.; Qin, D. Gold-Based Cubic Nanoboxes with Well-Defined Openings at the Corners and Ultrathin Walls Less Than Two Nanometers Thick. ACS Nano 2016, 10, 8019-8025. 
(5) Wang, Y.; Yan, B.; Chen, L. SERS Tags: Novel Optical Nanoprobes for Bioanalysis. Chem. Rev. 2013, 113, 1391-1428.

(6) Yang, Y.; Liu, J.; Fu, Z.-W.; Qin, D. Galvanic Replacement-Free Deposition of Au on Ag for Core-Shell Nanocubes with Enhanced Chemical Stability and SERS Activity. J. Am. Chem. Soc. 2014, 136, 8153-8156.

(7) Espinosa, A.; Curcio, A.; Cabana, S.; Radtke, G.; Bugnet, M.; Kolosnjaj-Tabi, J.; Péchoux, C.; Alvarez-Lorenzo, C.; Botton, G. A.; Silva, A. K. A.; Abou-Hassan, A.; Wilhelm, C. Intracellular Biodegradation of Ag Nanoparticles, Storage in Ferritin, and Protection by a Au Shell for Enhanced Photothermal Therapy. ACS Nano 2018, 12, 6523-6535.

(8) Mir-Simon, B.; Reche-Perez, I.; Guerrini, L.; Pazos-Perez, N.; Alvarez-Puebla, R. A. Universal One-Pot and Scalable Synthesis of SERS Encoded Nanoparticles. Chem. Mater. 2015, 27, 950-958.

(9) Shan, B.; Pu, Y.; Chen, Y.; Liao, M.; Li, M. Novel SERS Labels: Rational Design, Functional Integration and Biomedical Applications. Coord. Chem. Rev. 2018, 371, 11-37.

(10) Alvarez-Puebla, R. A.; Liz-Marzán, L. M. SERS-Based Diagnosis and Biodetection. Small 2010, 6, 604-610.

(11) Bodelón, G.; Montes-García, V.; Fernández-López, C.; Pastoriza-Santos, I.; Pérez-Juste, J.; Liz-Marzán, L. M. Au@pNIPAM SERRS Tags for Multiplex Immunophenotyping Cellular Receptors and Imaging Tumor Cells. Small 2015, 11, 4149-4157.

(12) Samanta, A.; Maiti, K. K.; Soh, K.-S.; Liao, X.; Vendrell, M.; Dinish, U. S.; Yun, S.-W.; Bhuvaneswari, R.; Kim, H.; Rautela, S.; Chung, J.; Olivo, M.; Chang, Y.-T. Ultrasensitive NearInfrared Raman Reporters for SERS-Based In Vivo Cancer Detection. Angew. Chem. Int. Ed. 2011, 50, 6089-6092.

(13) Wang, Y.; Schlücker, S. Rational Design and Synthesis of SERS Labels. Analyst 2013, 138, 2224-2238.

(14) Davis, R. M.; Kiss, B.; Trivedi, D. R.; Metzner, T. J.; Liao, J. C.; Gambhir, S. S. SurfaceEnhanced Raman Scattering Nanoparticles for Multiplexed Imaging of Bladder Cancer Tissue Permeability and Molecular Phenotype. ACS Nano 2018, 12, 9669-9679.

(15) Koo, K. M.; Wang, J.; Richards, R. S.; Farrell, A.; Yaxley, J. W.; Samaratunga, H.; Teloken, P. E.; Roberts, M. J.; Coughlin, G. D.; Lavin, M. F.; Mainwaring, P. N.; Wang, Y.; Gardiner, R. A.; Trau, M. Design and Clinical Verification of Surface-Enhanced Raman Spectroscopy Diagnostic Technology for Individual Cancer Risk Prediction. ACS Nano 2018, 12, 8362-8371. 
(16) Lenzi, E.; de Aberasturi, D. J.; Liz-Marzan, L. M. Surface-Enhanced Raman Scattering Tags for Three-Dimensional Bioimaging and Biomarker Detection. ACS Sens. 2019, 4, 11261137.

(17) Wen, S.; Miao, X.; Fan, G.-C.; Xu, T.; Jiang, L.-P.; Wu, P.; Cai, C.; Zhu, J.-J. AptamerConjugated Au Nanocage/ $/ \mathrm{SiO}_{2}$ Core-Shell Bifunctional Nanoprobes with High Stability and Biocompatibility for Cellular SERS Imaging and Near-Infrared Photothermal Therapy. ACS Sens. 2019, 4, 301-308.

(18) Sloan-Dennison, S.; Bevins, M. R.; Scarpitti, B. T.; Sauve, V. K.; Schultz, Z. D. Protein Corona-Resistant SERS Tags for Live Cell Detection of Integrin Receptors. Analyst 2019, 144, $5538-5546$.

(19) Lin, M.; Wang, Y. Q.; Sun, X. Y.; Wang, W. H.; Chen, L. X. "Elastic" Property of Mesoporous Silica Shell: For Dynamic Surface Enhanced Raman Scattering Ability Monitoring of Growing Noble Metal Nanostructures via a Simplified Spatially Confined Growth Method. ACS Appl. Mater. Interfaces 2015, 7, 7516-7525.

(20) Su, X. M.; Wang, Y. Q.; Wang, W. H.; Sun, K. X.; Chen, L. X. Phospholipid Encapsulated AuNR@Ag/Au Nanosphere SERS Tags with Environmental Stimulus Responsive Signal Property. ACS Appl. Mater. Interfaces 2016, 8, 10201-10211.

(21) Álvarez-Puebla, R. A.; Contreras-Cáceres, R.; Pastoriza-Santos, I.; Pérez-Juste, J.; LizMarzán, L. M. Au@pNIPAM Colloids as Molecular Traps for Surface-Enhanced, Spectroscopic, Ultra-Sensitive Analysis. Angew. Chem. Int. Ed. 2009, 48, 138-143.

(22) de Aberasturi, D. J.; Serrano-Montes, A. B.; Langer, J.; Henriksen-Lacey, M.; Parak, W. J.; Liz-Marzan, L. M. Surface Enhanced Raman Scattering Encoded Gold Nanostars for Multiplexed Cell Discrimination. Chem. Mater. 2016, 28, 6779-6790.

(23) Lim, D. K.; Jeon, K. S.; Hwang, J. H.; Kim, H.; Kwon, S.; Suh, Y. D.; Nam, J. M. Highly Uniform and Reproducible Surface-Enhanced Raman Scattering from DNA-Tailorable Nanoparticles with 1-nm Interior Gap. Nat. Nanotechnol. 2011, 6, 452-460.

(24) Kang, J. W.; So, P. T. C.; Dasari, R. R.; Lim, D. K. High Resolution Live Cell Raman Imaging Using Subcellular Organelle-Targeting SERS-Sensitive Gold Nanoparticles with Highly Narrow Intra-Nanogap. Nano Lett. 2015, 15, 1766-1772.

(25) Lin, L.; Zapata, M.; Xiong, M.; Liu, Z. H.; Wang, S. S.; Xu, H.; Borisov, A. G.; Gu, H. C.; Nordlander, P.; Aizpurua, J.; Ye, J. Nanooptics of Plasmonic Nanomatryoshkas: Shrinking the Size of a Core-Shell Junction to Subnanometer. Nano Lett. 2015, 15, 6419-6428. 
(26) Lin, L.; Gu, H. C.; Ye, J. Plasmonic Multi-Shell Nanomatryoshka Particles as Highly Tunable SERS Tags with Built-In Reporters. Chem Commun 2015, 51, 17740-17743.

(27) Wang, Y.; Wang, Y. Q.; Wang, W. H.; Sun, K. X.; Chen, L. X. Reporter-Embedded SERS Tags from Gold Nanorod Seeds: Selective Immobilization of Reporter Molecules at the Tip of Nanorods. ACS Appl. Mater. Interfaces 2016, 8, 28105-28115.

(28) Wang, Z. Y.; Zong, S. F.; Wu, L.; Zhu, D.; Cui, Y. P. SERS-Activated Platforms for Immunoassay: Probes, Encoding Methods, and Applications. Chem. Rev. 2017, 117, 79107963.

(29) Oldenburg, S. J.; Averitt, R. D.; Westcott, S. L.; Halas, N. J. Nanoengineering of Optical Resonances. Chem. Phys. Lett. 1998, 288, 243-247.

(30) Brinson, B. E.; Lassiter, J. B.; Levin, C. S.; Bardhan, R.; Mirin, N.; Halas, N. J. Nanoshells Made Easy: Improving Au Layer Growth on Nanoparticle Surfaces. Langmuir 2008, 24, 14166 14171.

(31) Shi, W. L.; Sahoo, Y.; Swihart, M. T.; Prasad, P. N. Gold Nanoshells on Polystyrene Cores for Control of Surface Plasmon Resonance. Langmuir 2005, 21, 1610-1617.

(32) Sauerbeck, C.; Haderlein, M.; Schurer, B.; Braunschweig, B.; Peukert, W.; Taylor, R. N. K. Shedding Light on the Growth of Gold Nanoshells. ACS Nano 2014, 8, 3088-3096.

(33) Skrabalak, S. E.; Chen, J.; Sun, Y.; Lu, X.; Au, L.; Cobley, C. M.; Xia, Y. Gold Nanocages: Synthesis, Properties, and Applications. Acc. Chem. Res. 2008, 41, 1587-1595.

(34) Polavarapu, L.; Liz-Marzán, L. M. Growth and Galvanic Replacement of Silver Nanocubes in Organic Media. Nanoscale 2013, 5, 4355-4361.

(35) Au, L.; Lu, X.; Xia, Y. A Comparative Study of Galvanic Replacement Reactions Involving Ag Nanocubes and $\mathrm{AuCl}_{2}^{-}$or $\mathrm{AuCl}_{4}^{-}$. Adv. Mater. 2008, 20, 2517-2522.

(36) Polavarapu, L.; Zanaga, D.; Altantzis, T.; Rodal-Cedeira, S.; Pastoriza-Santos, I.; PérezJuste, J.; Bals, S.; Liz-Marzán, L. M. Galvanic Replacement Coupled to Seeded Growth as a Route for Shape-Controlled Synthesis of Plasmonic Nanorattles. J. Am. Chem. Soc. 2016, 138, 11453-11456.

(37) Song, H.; Yang, Y.; Geng, J.; Gu, Z.; Zou, J.; Yu, C. Electron Tomography: A Unique Tool Solving Intricate Hollow Nanostructures. Adv. Mater. 2018, e1801564.

(38) Zhuo, X. L.; Henriksen-Lacey, M.; de Aberasturi, D. J.; Sanchez-Iglesias, A.; Liz-Marzan, L. M. Shielded Silver Nanorods for Bioapplications. Chem. Mater. 2020, 32, 5879-5889. 

Scattering with Nanoparticles. Acc. Chem. Res. 2016, 49, 2746-2755. Properties. Chem. Mater. 2014, 26, 2836-2846. Tomography. Ultramicroscopy 2015, 157, 35-47.

(40) Bastus, N. G.; Merkoci, F.; Piella, J.; Puntes, V. Synthesis of Highly Monodisperse Citrate-Stabilized Silver Nanoparticles of up to $200 \mathrm{~nm}$ : Kinetic Control and Catalytic

(41) van Aarle, W.; Palenstijn, W. J.; De Beenhouwer, J.; Altantzis, T.; Bals, S.; Batenburg, K. J.; Sijbers, J. The ASTRA Toolbox: A Platform for Advanced Algorithm Development in Electron 


\section{ToC graphic}

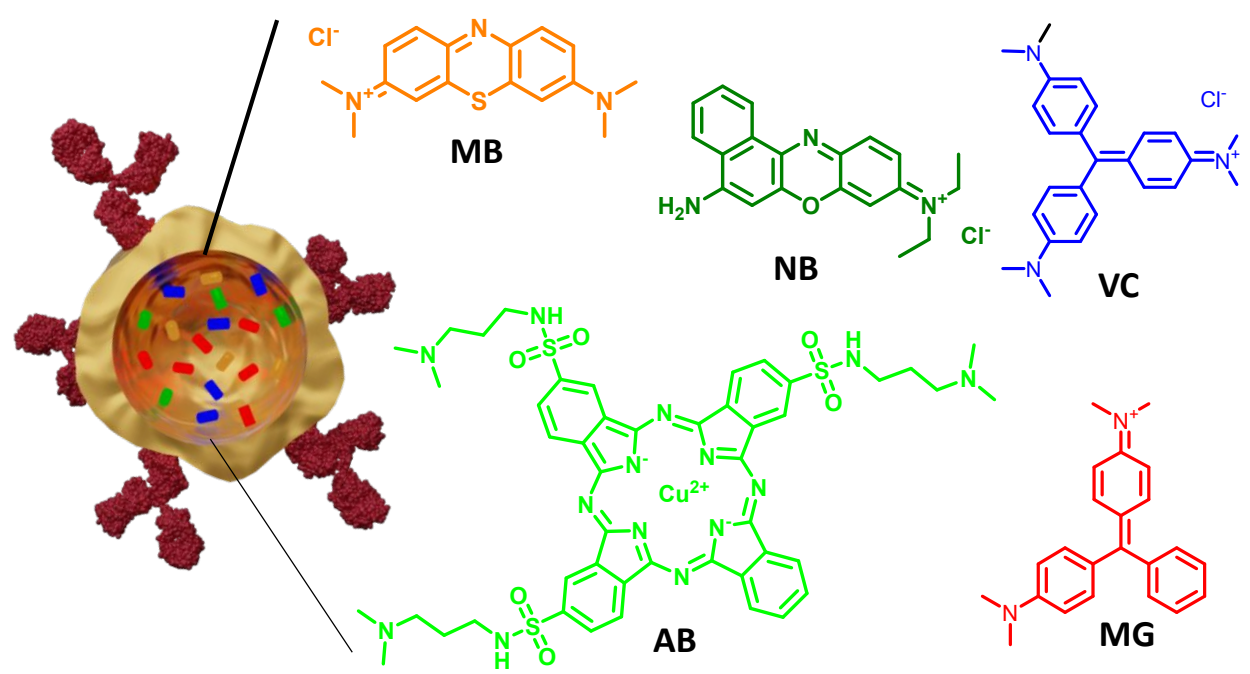

